\title{
Un acercamiento a la correlación entre habilidades tecnológicas de los docentes y su intención de uso de la tecnología en la enseñanza en el aula secondtitle
}

\author{
Heivar Yesid Rodriguez Pinzon ${ }^{\mathrm{a}}$ \\ Jhon Jairo Galvis Lopez ${ }^{\mathrm{b}}$ \\ heivarrodriguez@usantotomas.edu.co \\ Jhon.galvis@usantotomas.edu.co
}

\begin{abstract}
Resumen
En la búsqueda de encontrar La finalidad de este documento es utilizar un diseño de muestreo estratificado MAS a una población de 1724 de docentes de tiempo completo de tres universidades en Colombia (Tecnológica de Pereira, Quindío y Francisco José de Caldas), con el fin de presentar un modelo de ecuaciones estructurales basadas en la varianza SEM-PLS. El modelo establece cinco constructos (tres exógenos que corresponden a las creencias en las habilidades tecnológicas del docente y dos endógenos que corresponden a la intensión del uso de la tecnología en el aula) y 16 items o variables indicadoras, el instrumento y modelo usado se basa en el estudio previo propuesto por (Teo, 2009). Los resultados muestran que existe una relación positiva significativa entre la habilidad del uso de la tecnología para la pedagogía y la intención del uso constructivista y tradicional en el aula. Así mismo, el modelo de medida reflectivo muestra AVE mayores a 0.5 para los constructos, consistencia interna y fiabilidad mayor a $0.6 \mathrm{y}$ validez discriminante HTMT mediante intervalo de confianza que no incluya 1 en todos los constructos. Por otro lado, en el modelo estructural no se evidencia multicolinealidad, los coeficientes path o de trayectoria tienen magnitud fuerte y signo acorde a las hipótesis planteadas, en el caso del constructo TP (Tecnología para la pedagogía) hacia los constructos UCT (Intención del uso de la tecnología de forma constructivista) y UTT (Intención de uso de la tecnología de forma tradicional), el coeficiente de determinación de los constructos endógenos son mayores a 0.6 y se muestra un grado del constructo exógeno TP que explica los constructos endógenos UCT y UTT.
\end{abstract}

Palabras clave: SEM-PLS, ecuaciones estructurales, Modelo de medida, Modelo Estructural.

\begin{abstract}
The purpose of this document is to present a model of structural equations based on the SEM-PLS variance. The model establishes five constructs (three exogenous that correspond to the beliefs in the technological abilities of the teacher and two endogenous ones that correspond to the intention of the use of technology in the classroom) and 16 items, the instrument and model used is based on the previous study proposed by (Teo, 2009). The results show that there is a significant positive relationship between the ability to use technology for pedagogy and the intention of constructivist and traditional use in the classroom. Likewise, the reflective measurement model shows AVE greater than 0.5 for the constructs, internal consistency and reliability greater than 0.6 and HTMT discriminant validity through confidence interval that do not include 1 in all constructs. On the other hand, in the structural model there is no evidence of multicollinearity, path or trajectory coefficients have a strong magnitude and sign according
\end{abstract}

\footnotetext{
${ }^{a}$ Docente. Facultad de Estadística. Universidad Santo Tomas

Docente. Fundación Universitaria Konrad Lorenz

${ }^{\mathrm{b}}$ Estudiante.Maestría en Estadística Aplicada. Universidad Santo Tomas
} 
to the hypotheses, in the case of the construct TP (Technology for pedagogy) towards the constructs UCT (Intent to use the technology in a constructivist way) and UTT (intention to use technology in a traditional way), the coefficient determination of endogenous constructs are greater than 0.6 and a degree of the construct is shown exogenous TP that explains the endogenous constructs UCT and UTT.

Keywords: SEM-PLS, Structural Equations, Measurement Model, Structural Model .

\section{Introducción}

El presente estudio nace de la necesidad de abordar el análisis de las creencias del docente hacia el uso de las tecnologías en el aula. Distintos autores e investigadores han propuesto investigaciones que intenten explicar este fenómeno (Barclay, Higgins, Thompson, 1995; Chiu Churchill, 2016; Estévez-Nenninger, Valdés-Cuervo, Arreola-Olivarria, Zavala-Escalante, 2014; Jääskelä, Häkkinen, Rasku-Puttonen, 2017a; Jimoyiannis Komis, 2007; Lawrence Lentle-Keenan, 2013; Lumpe Chambers, 2001; Teo, 2009) y mediante distintos métodos estadísticos y metodologías de investigación han encontrado que el docente puede tener creencias sobre su eficacia o habilidad en el uso de las tecnologías en el aula. Sin embargo, estudios similares usando ecuaciones estructurales en Colombia no se han propuesto, lo cual es una oportunidad de presentar una propuesta que permita explorar este tema usando técnicas de muestreo para determinar el tamaño muestral y abordar mediante ecuaciones estructurales basadas en la varianza, un modelo que relacione las creencias de las habilidades en el uso de las tecnologías y la intención del uso de la tecnología en el aula.

El estudio inicia con una definición del problema, el cual plantea una pregunta general, la cual mediante una conceptualización teórica, se propone un diseño muestral para estimar el tamaño de la muestra objetivo utilizando el diseño Estratificado-MAS y con la información obtenida del instrumento adaptado de (Teo, 2009), se recoge la información necesaria para desarrollar el modelo SEM-PLS que permita falsear seis hipótesis y encontrar la posible relación entre las creencias de las habilidades del uso de las tecnologías y la intención del uso de las tecnologías en el aula.

En García, Castillo, Ortíz (2015) se menciona que tendencias como infraestructuras habilitadoras apoyadas por las tecnologías facilitaran los procesos de aprendizaje, es decir, la interacción de herramientas como el internet, la computación en la nube, el uso de dispositivos móviles, entre otros, jugarán un rol significativo en los procesos formativos actuales. Por ello, la enseñanza tradicional en la cual se basa en un proceso jerárquico profesor alumno, ya está siendo reconsiderada con el uso educativo de las tecnologías de la información TIC's (Andrews Haythornthwaite, 2007), lo que plantea nuevos paradigmas, por ejemplo: cómo la forma de enseñar del profesor actual se relaciona con el uso de las tecnologías, cuáles deben ser sus competencias, qué rol debe jugar en el escenario de la educación virtual, entre otras. Sin embargo, para tratar estos paradigmas, es necesario iniciar con conocer cómo las creencias del uso de la tecnología en el aula son asumidas por los docentes.

Ertmer Ottenbreit-Leftwich (2010) mencionan que los docentes con bajo nivel de uso de la tecnología no deberían interactuar con estudiantes con alto manejo tecnológico, ya que el proceso formativo podría fallar y sugieren que es necesario conocer y gestionar los cambios de cultura, creencias y conocimientos hacia el uso de la tecnología en la enseñanza por parte de los docentes.

En este sentido, Teo (2009) estudió la relación entre la creencia del estudiante de licenciatura y el uso eficaz de la tecnología para la enseñanza mediante una aproximación de ecuaciones estructurales basadas en la covarianza, lo cual, aportó al entendimiento de la relación que existe entre las creencias del estudiante de licenciatura y su capacidad para usar la tecnología y cómo ellos deberían utilizar la tecnología en la enseñanza. Así mismo, se han estudiado distintos tipos de creencias y cómo ellas evolucionan cuando los docentes adquieren experiencia (Estévez-Nenninger et al., 2014), es decir, a medida que el docente se acerca a nuevas formas de enseñar, su pensamiento tradicional de enseñanza cambia 
acorde al nuevo proceso cognitivo que el docente va afrontando.

Autores como Chiu Churchill (2016) han realizado investigaciones de cómo las creencias, actitudes y ansiedades en la enseñanza se ven afectadas al usar tecnologías con dispositivos móviles en el aula. Sus resultados mostraron que las creencias cambian y la actitud es positiva hacia el uso de dispositivos móviles en el aula, sin embargo, la ansiedad se mantiene en la mayoría de los docentes del estudio al usar nuevas tecnologías. Así mismo, a medida que los docentes usan la tecnología móvil, la ansiedad disminuye con el paso del tiempo. Por otro lado, dado que específicamente en Colombia no se han realizado estudios sobre las creencias del uso de la tecnología de los docentes en educación superior y en condiciones actuales, las instituciones están invirtiendo recursos y tiempo en desarrollar currículos con integración de tecnologías de la información (Álvarez, 2002), lo cual, invita a plantear estudios de cómo las creencias de los docentes afectan el proceso de articulación con la tecnología, por lo tanto, esta propuesta de grado busca estudiar la relación entre las creencias de las habilidades tecnológicas del docente y la intención del uso de la tecnología en la enseñanza en el aula.

Por otro lado, se apoya la presente investigación en las teorías de muestreo (Särndal, Swensson, Wretman, 2003) y las ecuaciones estructurales basadas en la varianza (Hair, 2017). Por lo tanto, se plantea la siguiente pregunta general: ¿Cuál es la relación entre las creencias de las habilidades tecnológicas del docente y la intención del uso de la tecnología en la enseñanza en el aula de las universidades Tecnológica de Pereira, Quindío y Francisco José de Caldas?

Jääskelä et al., (2017b) realizan un estudio cualitativo en donde encuentran una gran variedad de creencias de los docentes, entre ellas, que éstas varían según el pensamiento pedagógico de cada docente y que la adopción del uso de la tecnología está condicionado por presiones externas, lo cual, cuando sucede esto, a largo plazo el docente no piensa en los propósitos educativos y no ocurre una expectativa de cambio en la pedagogía. Otro aspecto resultante, es la definición del valor de la tecnología como la relación tanto con la pedagogía deseada o la intencionalidad del uso de la tecnología, como con el propio trabajo de los docentes. A modo de futuros trabajos, los autores proponen "Dado que los docentes son actores clave en el desarrollo de la enseñanza, es necesario realizar más estudios para obtener pruebas sobre la influencia de las creencias de los docentes en su uso de la tecnología en las prácticas pedagógicas", p.12. Así mismo, Chiu Churchill (2016) afirman que "los maestros que perciben una mayor autoeficacia en el uso de la computadora tienen más probabilidades de encontrar nuevas tecnologías fáciles de usar y útiles en la enseñanza, y tienden a adoptar las tecnologías en la enseñanza", p.3. Luego, la propuesta de investigación que se presenta es significativa, ya que busca presentar una la relación entre las creencias de las habilidades tecnológicas del docente y la intención del uso de la tecnología en la enseñanza en el aula de las universidades Tecnológica de Pereira, Quindío y Francisco José de Caldas. De los posibles resultados obtenidos, se podrá proponer estudios futuros sobre el diseño de estrategias apropiadas para mejorar las habilidades del uso de la tecnología en la enseñanza en el aula de los docentes y que factores podrían causar el cambio de esas creencias.

Las creencias son las ideas con las que las personas se comprometen, a veces llamadas valores centrales (Lumpe Chambers, 2001) y se han estudiado en el uso de la tecnología en educación usando la teoría de la autoeficacia de Bandura, el cual define "la autoeficacia como las creencias en las capacidades de uno para organizar y ejecutar los cursos de acción requeridos para producir los logros dados" (2001, p. 93). Chiu Churchill (2016) mencionan que "las creencias son fundamentales para aceptar nuevas tecnologías e incluir su visión en la adquisición de nuevo conocimiento y maneras efectivas de enseñar", p.2. Por otro lado, Devine, Fahie, McGillicuddy (2013) afirman que "las creencias también se entrelazan con las identidades y se centran en ellas: cómo los maestros se definen a sí mismos y cómo entienden y definen a los demás".

Una propuesta de investigación que busca relacionar la creencias de la autoeficacia o de las habilidades en el uso de las tecnologías y uso intencionalidad para la educación, es dada por (Teo, 2009) que amplía el estudio previo realizado por él de la creencia en la enseñanza constructivista y el uso de la 
tecnología en 2008. Dentro de sus resultados se encuentran una evidencia empírica de la relación significativa del uso de la tecnología y la intencionalidad de uso de la misma en el aula pero aplicada a estudiantes de licenciatura.

\section{Material y métodos}

Moreno Torres (1993) define la muestra aleatoria estratificada como "cuando se separa los elementos de una población en grupos en grupos mutuamente excluyentes (un elemento de la población pertenece a uno y solo uno de los grupos) y conjuntamente exhaustivos (la unión de todos los elementos de los grupos es igual a la población total)", p. 161. El muestreo estratificado suministra una cantidad específica de información a menor costo que el muestreo aleatorio simple (Pérez López, 2014) y se recomienda cuando la población está conformada en un conjunto de grupos heterogéneos entre sí y homogéneos dentro de ellos (Chambers Clark, 2012; Gutiérrez, 2009). Se da la estratificación cuando se conoce una variable auxiliar, por ejemplo, el lugar de nacimiento, el nivel de educación, etc.

En cada estrato es posible aplicar un diseño muestral, en el caso de afijación proporcional, se asume que el tamaño de cada estrato es conocido y al igual el tamaño de la población (Särndal et al., 2003); Pérez López (2014) asegura que "las fracciones de muestreo en los estratos son iguales y coinciden con la fracción global de muestreo, siendo su valor la constante de proporcionalidad.", p. 175. Posteriormente, se realiza un muestreo aleatorio simple MAS en cada estrato. Lo que se busca finalmente en el estratificado MAS es lograr una mayor representatividad de cada estrato al darle mayor probabilidad de aparecer en la muestra según el tamaño de cada uno y cumpliendo el supuesto de aleatoriedad al aplicar un MAS en cada estrato.

Barclay et al. (1995) define el modelamiento de ecuaciones estructurales como "la forma en que se evalúa simultáneamente teorías que involucran constructos (no observables o variables latentes) con sus múltiples medidas observables y la estimación de la relación entre esos constructos" , p.287.

El modelamiento de ecuaciones estructurales basadas en covarianza divide la varianza de cada indicador en dos partes, la varianza común y la varianza única. La varianza común se estima a partir de la varianza compartida con otros indicadores en el modelo de medición del constructo (Hair, 2014). La varianza única consiste en la varianza específica dada por cada ítem, la cual es sistemática y confiable y que contiene un componente de varianza del error, del cual se supone es aleatoria y no confiable.

En el modelamiento de ecuaciones estructurales basadas en varianza o SEM-PLS, no se divide la varianza en dos partes, el objetivo es mostrar la varianza total en los indicadores observados en lugar de explicar solo las correlaciones entre los individuos (Kwong Kwong, 2013). Con respecto a la muestra requerida, SEM-PLS ha tenido bastantes puntos de vista (Sarstedt, Hair, Ringle, Thiele, Gudergan, 2016), pero Reinartz et al., (2009) estima como resultado de una investigación que realizó que una tamaño muestral mayor a 100 observaciones puede ser suficiente para que exista un nivel aceptable de poder estadístico (probabilidad de rechazar correctamente la hipótesis nula cuando debe ser rechazada). Así mismo, cuando el enfoque de la investigación es exploratoria se puede usar SEM-PLS, en la cual, se hace una rápida identificación de relaciones potenciales entre variables y se presta atención a la magnitud de los caminos estructurales, que a su vez, posteriormente se necesite una investigación de tipo casual (Henseler, 2018).

En la figura 1 se puede apreciar un ejemplo de un modelo de sistemas de ecuaciones estructurales, el cual tiene dos partes: 1.) El modelo de medida y 2.) El modelo estructural. 


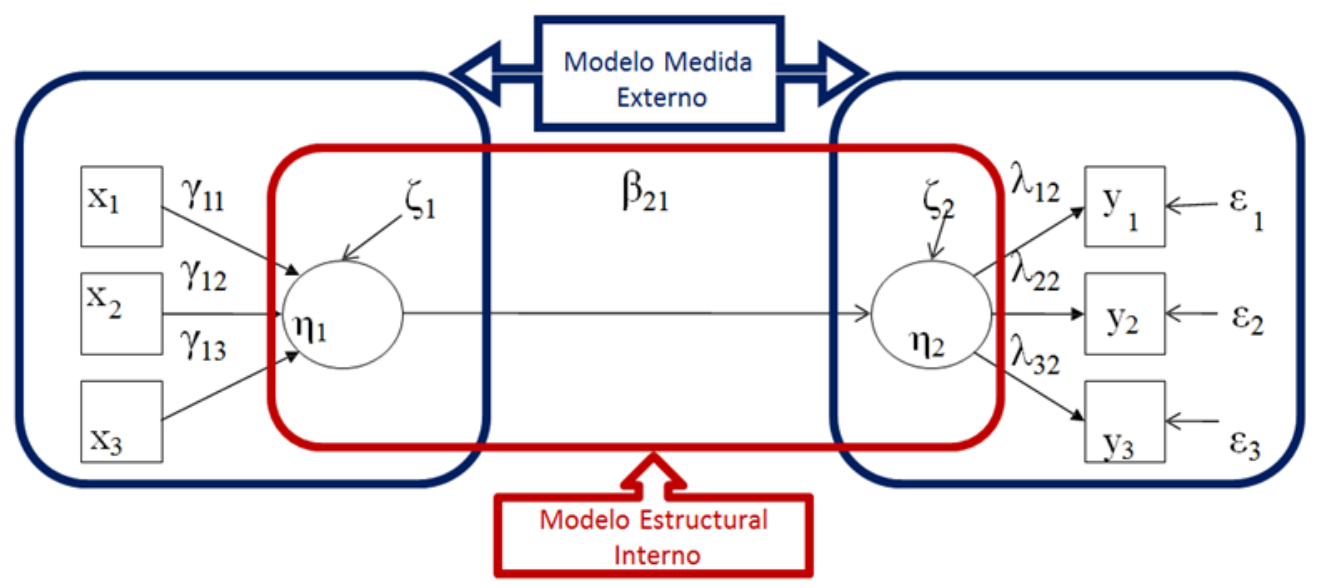

Figura 1: Modelo de ecuaciones estructurales

En el modelo de medida se muestra las relaciones entre los constructos y sus variables indicadoras, estas relaciones están definidas condicionalmente por una teoría de medida y las hipótesis son planteadas a partir de esas relaciones (Hair, 2014, p. 44). Existen dos tipos de modelos de medida, los reflectivos (pesos de correlación) y formativos (pesos de regresión). El modelo de medida reflectivo, las flechas direccionales van del constructo a los indicadores y en el modelo de medida formativo, las flechas direccionales van de las variables indicadoras al constructo. Sin embargo, en el modelo de medida formativo, investigadores actuales han distinguido dos tipos variables indicadoras: compuestas y casuales (Hair, 2014; Sarstedt et al., 2016). Las variables indicadoras compuestas forman el constructo y las causales lo causan. La escogencia de un modelo de medida depende de la teoría y del investigador, ya que un mismo constructo puede tener distintas interpretaciones según la disciplina en la que se evalué (Sarstedt et al., 2016).

En el modelo estructural se propone las relaciones entre las variables latentes o constructos, estas relaciones también permite plantear hipótesis soportadas por alguna teoría.

Entre las recomendaciones para el análisis en SEM-PLS que se deben tener en cuenta en el modelo de medida reflectiva son (Aldás Manzano Uriel Jiménez, 2017, p. 613; Hair, 2017, p. 106):

- Consistencia interna y fiabilidad: La consistencia interna es una forma de fiabilidad usada para juzgar la consistencia de los resultados sobre los ítems en el instrumento (Hair, 2017, p. 314). Esto determina sí los ítems que miden un constructo son similares en sus puntajes (ejemplo, correlación entre ítems es mayor). Para la fiabilidad compuesta y alfa de Cronbach se proponen valores mayores a 0.70 .

- Validez convergente: Es el grado en que un constructo de medida formativa se correlaciona positivamente con una medida alternativa (reflectivo o un item) del mismo constructo (Hair, 2017, p. 315). Las cargas deben ser significativas y superiores a 0.70. La varianza extraída promedio AVE mayor a 0.50 .

- Validez discriminante (AVE y/o HTMT): Mide sí los constructos son diferentes o no. La AVE de cada variable latente debe ser mayor al cuadrado de la correlación más grande que esa variable latente tenga con cualquier otra variable latente. Para HTMT (Heterotrait-monotrait ratio) es un método más riguroso para muestras pequeñas y su valor no deber ser igual o mayor a 0.9.

- Para las pruebas de hipótesis, si bien SEM-PLS es una técnica no paramétrica, se puede usar boostraping para estimar la significancia de las relaciones a través del re muestreo (Gutiérrez, 2009; Hair, 2017). 
Entre las recomendaciones para el análisis en SEM-PLS que se deben tener en cuenta en el modelo estructural son (Aldás Manzano Uriel Jiménez, 2017, p. 613; Hair, 2017, p. 222):

- Evaluación de multicolinealidad VIF: Al igual que en regresión lineal, se debe evaluar la alta correlación entre variables antecedentes de cada uno de los constructos endógenos. Se recomienda un valor menor a $\mathrm{VIF}<5$ con una tolerancia menor de 0.2 .

- Evaluación de la magnitud de los coeficientes path o de trayectoria: Aquella trayectoria cuyo signo sea distinto al signo postulado en la hipótesis, se declara no soportada. Los valores están dentro +1 o -1 (aunque pueden ser un poco menores o mayores a estas fronteras), y los valores cercanos a cero son habitualmente no significativos.

- Establecer la significancia de las relaciones mediante boostraping: El bootstraping permite evaluar si las relaciones son significativamente diferentes de cero, y consiste en un muestreo repetitivo aleatorio con reposición de la muestra original. El resultado de este proceso muestra el error estándar, t estadístico y los intervalos de confianza de los parámetros. El número de sub muestras debe ser igual al menos al número de observaciones usadas en el modelo (Hair, 2017). Cuando se formula la hipótesis una relación + o - entre variables, se utiliza la distribución t de una cola con n-1 grados de libertad, donde $n$ es el número de sub muestras.

- Evaluación del coeficiente de determinación R2: Muestra la cantidad de varianza de un constructo que es explicada por las variables predictoras de dicho constructo endógeno. Se determina que un valor 0.75 como sustancial, 0.5 moderado y 0.25 débil (Hair, 2017, p. 222).

- Tamaño del efecto f2: Valora el grado con el que un constructo exógeno contribuye a explicar un determinado constructo endógeno en términos del coeficiente de determinación. Valores de 0.02, 0.15 y 0.35 son interpretados como pequeños, medianos y grandes.

\subsection{Diseño del instrumento}

El diseño del instrumento es la adaptación del propuesto por (Teo, 2009) y la operacionalización de variables se fundamentan en dicha propuesta, la cual realiza una justificación conceptual y teórica cuando es presentado el instrumento. La siguiente tabla presenta el instrumento propuesto adaptado a partir de (Teo, 2009).

\begin{tabular}{|c|c|c|c|}
\hline Dimensión & Indicadores & Item / variable & Unidad de medida \\
\hline $\begin{array}{l}\text { Creencias de } \\
\text { las habilidades } \\
\text { del uso de las } \\
\text { tecnologías }\end{array}$ & $\begin{array}{l}\text { Habilidades } \\
\text { Básicas } \\
\text { Tecnológicas } \\
\text { (HTB) }\end{array}$ & $\begin{array}{l}\text { HTB1: Puedo usar Internet para } \\
\text { buscar información y recursos } \\
\text { educativos. } \\
\text { HTB2: Puedo usar el procesador } \\
\text { de textos para crear, editar y } \\
\text { organizar documentos para fines } \\
\text { específicos. ( Por ejemplo, } \\
\text { Microsoft Word u otro) HTB3: } \\
\text { Puedo hacer presentaciones } \\
\text { usando software de Presentación } \\
\text { para el aula (por ejemplo, } \\
\text { Microsoft Powerpoint u otro) }\end{array}$ & $\begin{array}{l}\text { Escala tipo Likert de } 1-7 . \\
\text { 1: Totalmente de acuerdo } \\
\text { 2: De acuerdo } \\
\text { 3: Parcialmente de acuerdo } \\
\text { 4: Ni de acuerdo ni en desacuerdo } \\
\text { 5: Parcialmente en desacuerdo } \\
\text { 6: En desacuerdo } \\
\text { 7: Totalmente en desacuerdo }\end{array}$ \\
\hline
\end{tabular}




\begin{tabular}{|c|c|c|c|}
\hline Dimensión & Indicadores & Item / variable & Unidad de medida \\
\hline \multirow[b]{2}{*}{$\begin{array}{l}\text { Creencias de } \\
\text { las habilidades } \\
\text { del uso de las } \\
\text { tecnologías }\end{array}$} & $\begin{array}{l}\text { Habilidades } \\
\text { Avanzadas } \\
\text { Tecnológicas } \\
\text { (HTA) }\end{array}$ & $\begin{array}{l}\text { HTA1: Puedo usar editores de } \\
\text { sitios web para crear y / o } \\
\text { modificar páginas web (por } \\
\text { ejemplo, Microsoft FrontPage, } \\
\text { Macromedia Dreamweaver u otro) } \\
\text { HTA2: Puedo usar software de } \\
\text { edición de video (por ejemplo, } \\
\text { Microsoft Movie Maker, Adobe } \\
\text { Premiere, Ulead Video Studio, u } \\
\text { otro) } \\
\text { HTA3: Puedo usar software de } \\
\text { animación (por ejemplo, } \\
\text { Macromedia Flash, Authorware, } \\
\text { Director u otro) para crear } \\
\text { animaciones. }\end{array}$ & $\begin{array}{l}\text { Escala tipo Likert de 1-7. } \\
\text { 1: Totalmente de acuerdo } \\
\text { 2: De acuerdo } \\
\text { 3: Parcialmente de acuerdo } \\
\text { 4: Ni de acuerdo ni en desacuerdo } \\
\text { 5: Parcialmente en desacuerdo } \\
\text { 6: En desacuerdo } \\
\text { 7: Totalmente en desacuerdo }\end{array}$ \\
\hline & $\begin{array}{l}\text { Habilidades del } \\
\text { uso de la } \\
\text { Tecnología para } \\
\text { La Pedagogía } \\
\text { (TP) }\end{array}$ & $\begin{array}{l}\text { TP1: Busco, evalúo y selecciono } \\
\text { los recursos de tecnologías de la } \\
\text { información apropiados para } \\
\text { apoyar las actividades de clase } \\
\text { TP2: Soy capaz de acoger y } \\
\text { aplicar actividades de aprendizaje } \\
\text { basadas en tecnologías de la } \\
\text { información en el aula } \\
\text { TP3: Puedo gestionar actividades } \\
\text { de aprendizaje basadas en } \\
\text { tecnologías de la información en } \\
\text { un laboratorio informático } \\
\text { TP4: Puedo acoger y aplicar } \\
\text { actividades que incorporan el uso } \\
\text { de las tecnologías de la } \\
\text { información para evaluar el } \\
\text { aprendizaje de los alumnos y } \\
\text { proporcionar retroalimentación } \\
\text { inmediata y constructiva }\end{array}$ & $\begin{array}{l}\text { Escala tipo Likert de 1-7. } \\
\text { 1: Totalmente de acuerdo } \\
\text { 2: De acuerdo } \\
\text { 3: Parcialmente de acuerdo } \\
\text { 4: Ni de acuerdo ni en desacuerdo } \\
\text { 5: Parcialmente en desacuerdo } \\
\text { 6: En desacuerdo } \\
\text { 7: Totalmente en desacuerdo }\end{array}$ \\
\hline $\begin{array}{l}\text { Uso intencional } \\
\text { de las } \\
\text { tecnologías en } \\
\text { el aula }\end{array}$ & $\begin{array}{l}\text { Uso de la } \\
\text { tecnología de } \\
\text { forma } \\
\text { tradicional en el } \\
\text { aula (UTT) }\end{array}$ & $\begin{array}{l}\text { UTT1: En mi clase, uso la } \\
\text { tecnología para enseñar a mi } \\
\text { alumno a dominar las habilidades } \\
\text { que desarrollo en clase } \\
\text { UTT2: En mi clase, utilizo la } \\
\text { tecnología para enseñar a mi } \\
\text { alumno a fortalecer conceptos } \\
\text { UTT3: En mi clase, uso la } \\
\text { tecnología para enseñar a mi } \\
\text { estudiante a practicar con } \\
\text { preguntas de opción múltiple }\end{array}$ & $\begin{array}{l}\text { Escala tipo Likert de 1-7. } \\
\text { 1: Totalmente de acuerdo } \\
\text { 2: De acuerdo } \\
\text { 3: Parcialmente de acuerdo } \\
\text { 4: Ni de acuerdo ni en desacuerdo } \\
\text { 5: Parcialmente en desacuerdo } \\
\text { 6: En desacuerdo } \\
\text { 7: Totalmente en desacuerdo }\end{array}$ \\
\hline
\end{tabular}




\begin{tabular}{|c|c|c|c|}
\hline Dimensión & Indicadores & Item / variable & Unidad de medida \\
\hline $\begin{array}{l}\text { Uso intencional } \\
\text { de las } \\
\text { tecnologías en } \\
\text { el aula }\end{array}$ & $\begin{array}{l}\text { Uso de la } \\
\text { tecnología de } \\
\text { forma } \\
\text { constructivista } \\
\text { en el aula } \\
\text { (UCT) }\end{array}$ & $\begin{array}{l}\text { UCT1: En mi clase, utilizo la } \\
\text { tecnología para enseñar a mi } \\
\text { alumno a trabajar de forma } \\
\text { colaborativa con los compañeros. } \\
\text { UCT2: En mi clase, uso la } \\
\text { tecnología para enseñar a mi } \\
\text { alumno a trabajar de forma } \\
\text { autónoma UCT3: En mi clase, } \\
\text { uso la tecnología para enseñar a } \\
\text { mi alumno a encontrar ideas e } \\
\text { información por sí solo }\end{array}$ & $\begin{array}{l}\text { Escala tipo Likert de } 1-7 . \\
\text { 1: Totalmente de acuerdo } \\
\text { 2: De acuerdo } \\
\text { 3: Parcialmente de acuerdo } \\
\text { 4: Ni de acuerdo ni en desacuerdo } \\
\text { 5: Parcialmente en desacuerdo } \\
\text { 6: En desacuerdo } \\
\text { 7: Totalmente en desacuerdo }\end{array}$ \\
\hline
\end{tabular}

Tabla 1: Diseño del instrumento y operacionalización de variables Fuente: Adaptado a partir de (Teo, 2009)

\subsection{Definición de la población y muestra}

La población objetivo está conformada por las siguientes universidades: Tecnológica de Pereira, Quindío y Francisco José de Caldas. El tamaño de la muestra se define tomando como referencia el tamaño de la población de 1724 docentes de tiempo fijo de las tres universidades objeto del estudio de la base de datos del total de docentes de educación superior vinculados laboralmente en Colombia al año 2016 del Ministerio de Educación Nacional. La tabla 2, muestra estratificación de los docentes de las tres universidades en el estudio. En el caso de la Universidad del Quindío el total reportado según el Ministerio de Educación Nacional al año 2016 es de 457 docentes de tiempo fijo, 501 para la Universidad Tecnológica de Pereira y 766 para la Universidad Distrital. La afijación proporcional estimada para cada estrato del total se representa con $27 \%$ para la Universidad del Quindío, $29 \%$ para la Tecnológica de Pereira y $44 \%$ para la Universidad Distrital. El tamaño muestral se puede ver en la tabla 3, la muestra tendrá un tamaño de 101 observaciones. Para este estudio no se considera un valor de muestra bajo, ya que en el uso del SEM-PLS se sugiere observaciones mayores a 100 (Reinartz et al., 2009).

\begin{tabular}{|l|l|c|}
\hline IES & Docentes Tiempo fijo & $\%$ \\
\hline Universidad del Quindío & 457 & $27 \%$ \\
\hline Universidad Tecnológica de Pereira & 501 & $29 \%$ \\
\hline Universidad Distrital & 766 & $44 \%$ \\
\hline Total Docentes & $\mathbf{1 7 2 4}$ & \multicolumn{1}{|l}{} \\
\cline { 1 - 2 } & &
\end{tabular}

Tabla 2: Características de la Población estratificada y la afijación proporcional

Fuente: Elaboración propia

\begin{tabular}{|l|l|}
\hline N Población & 1724 \\
\hline Confianza & 0,9 \\
\hline$Z^{2}$ & 2,71 \\
\hline $\mathbf{P}$ & 0,5 \\
\hline Margen de error & Tamaño muestra \\
\hline 0,01 & 1375 \\
\hline 0,02 & 855 \\
\hline 0,03 & 524 \\
\hline 0,04 & 340 \\
\hline
\end{tabular}




\begin{tabular}{|l|l|}
\hline 0,05 & 235 \\
\hline 0,06 & 170 \\
\hline 0,07 & 129 \\
\hline $\mathbf{0 , 0 8}$ & $\mathbf{1 0 1}$ \\
\hline
\end{tabular}

Tabla 3: Estimación del tamaño muestral

\subsection{Definición de hipótesis}

Se proponen las siguientes hipótesis nulas

- Ho1: No existe una la relación positiva significativa entre la creencia del manejo básico de la tecnología y el uso para la enseñanza en forma tradicional en el aula

- Ho2: No existe una la relación positiva significativa entre la creencia del manejo avanzado de la tecnología y el uso para la enseñanza en forma tradicional en el aula

- Ho3: No existe una la relación positiva significativa entre la creencia del manejo básico de la tecnología y el uso para la enseñanza en forma constructivista en el aula

- Ho4: No existe una la relación positiva significativa entre la creencia del manejo avanzado de la tecnología y el uso para la enseñanza en forma constructivista en el aula

- Ho5: No existe una la relación positiva significativa entre la creencia en su capacidad de usar la tecnología para la pedagogía y el uso para la enseñanza en forma tradicional en el aula

- Ho6: No existe una la relación positiva significativa entre la creencia en su capacidad de usar la tecnología para la pedagogía y el uso para la enseñanza en forma constructivista en el aula

\subsection{Definición del modelo propuesto}

La figura 2, muestra el modelo propuesto y adaptado de (Teo, 2009) para el análisis propuesto. Los constructos de estudio son dos y cada uno está compuesto por una serie de constructos que lo definen. El primer constructo: Las creencias de las habilidades tecnológicas del docente y los constructos que lo componente son: a) TP: Tecnología para la Pedagogía, b) HTB: Uso básico de la Tecnología y c) HTA: Uso avanzado de la Tecnología. El segundo constructo: d) UTT: Uso tradicional de la Tecnología y e) UCT: Uso constructivista de la Tecnología 


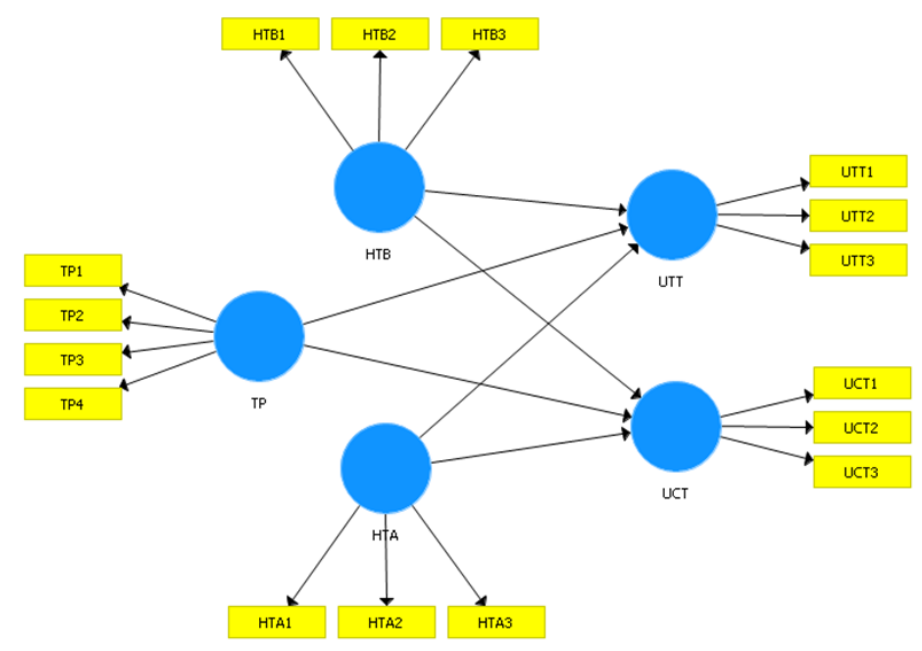

Figura 2: Modelo SEM-PLS propuesto

Fuente: Elaboración propia en SmartPLS 3.0

\section{Análisis y resultados}

El análisis se realiza en el Software SmartPLS 3.0 versión profesional con limitación de uso de 30 días. Inicialmente, se evalúa los datos, no hay presencia de datos perdidos. La edad media de los participantes es de 41 años, la edad mínima es de 28 años y máxima de 71 años. Se desarrolla en análisis de medida y estructural del modelo propuesto.

\subsection{Analisis de resultados modelo de medida}

La figura 3, muestra el resultado que arroja el software SmartPLS, el resumen de los valores relevantes para el analisis se puede observar en la tabla 5. En la Tabla 4, se muestra el resultado de la prueba HTMT, la cual permite analizar la validez discriminante y que tiene como criterio que ninguno de los intevalos de confianza posean un valor de 1 o mayor

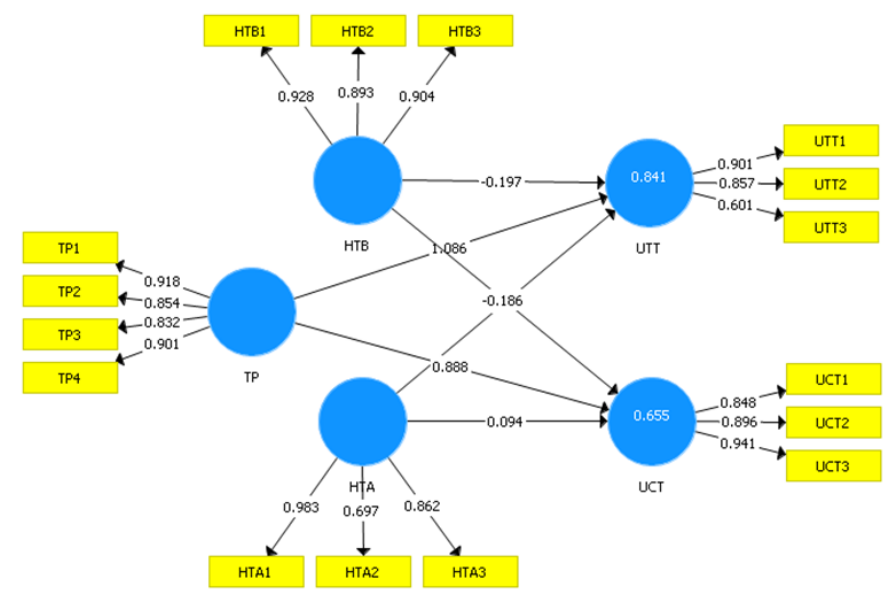

Figura 3: Resultado del modelo en SmartPLS 


\begin{tabular}{|l|l|l|}
\hline Variable/IC & $\mathbf{2 . 5} \%$ & $\mathbf{9 7 . 5} \%$ \\
\hline HTB $->$ HTA & 0.325 & 0.877 \\
\hline TP $->$ HTA & 0.336 & 0.849 \\
\hline TP $->$ HTB & 0.537 & 0.899 \\
\hline UCT $->$ HTA & 0.239 & 0.780 \\
\hline UCT $->$ HTB & 0.322 & 0.775 \\
\hline UCT $->$ TP & 0.635 & 0.909 \\
\hline UTT $->$ HTA & 0.226 & 0.748 \\
\hline UTT $->$ HTB & 0.402 & 0.838 \\
\hline UTT $->$ TP & 0.816 & 0.992 \\
\hline UTT $->$ UCT & 0.766 & 0.956 \\
\hline
\end{tabular}

Tabla 4: Resultados del bootstraping prueba HTMT Fuente: Elaboración propia

Los resultados dados por la tabla 5 permiten inferir que el modelo de medida reflectivo asegura la fiabilidad y validez de las medidas de los constructos y apoya la factibilidad de incluirlas en el modelo. Como se puede apreciar, la validez del discriminante asegura que cada constructo reflectivo comparte más varianza con sus propias variables indicadoras que con otros constructos en el modelo. Cada resultado es comparado con unos valores mínimos que son propuestos por la literatura (Hair, 2017), por ejemplo, un valor aceptable de AVE mayor a 0.50 .

\begin{tabular}{|c|c|c|c|c|c|c|c|}
\hline \multirow{3}{*}{$\begin{array}{l}\text { Latente } \\
\text { variable }\end{array}$} & \multirow{3}{*}{$\begin{array}{l}\text { Variables } \\
\text { Indicadoras }\end{array}$} & \multicolumn{3}{|c|}{ Validez convergente } & \multicolumn{2}{|c|}{$\begin{array}{l}\text { Consistencia Interna } \\
\text { y fiabilidad }\end{array}$} & \multirow{2}{*}{$\begin{array}{l}\text { Validez } \\
\text { discriminante }\end{array}$} \\
\hline & & Cargas & $\begin{array}{l}\text { Indicador } \\
\text { fiabilidad }\end{array}$ & AVE & $\begin{array}{l}\text { Fiabilidad } \\
\text { compuesta }\end{array}$ & $\begin{array}{l}\text { Alfa de } \\
\text { Cronbach }\end{array}$ & \\
\hline & & $>0,70$ & $>0,50$ & $>0,5$ & $>0,60$ & $>0,60$ & $\begin{array}{l}\text { HTMT Intervalo } \\
\text { de confianza que } \\
\text { no incluir } 1 \text {, ver } \\
\text { tabla } 3\end{array}$ \\
\hline \multirow{4}{*}{$\mathrm{TP}$} & TP1 & 0,918 & 0,842 & \multirow{4}{*}{0,732} & \multirow{4}{*}{0,890} & \multirow{4}{*}{0,889} & \multirow[t]{4}{*}{ 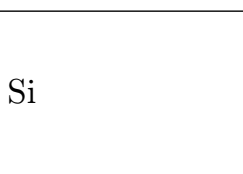 } \\
\hline & TP2 & 0,854 & 0,729 & & & & \\
\hline & TP3 & $\begin{array}{c}0,832 \\
\end{array}$ & $\begin{array}{l}0,692 \\
\end{array}$ & & & & \\
\hline & TP4 & 0.901 & 0,811 & & & & \\
\hline \multirow{3}{*}{ HTB } & HTB1 & 0,928 & 0,861 & \multirow{3}{*}{0,826} & \multirow{3}{*}{0,934} & \multirow{3}{*}{0,934} & \multirow{3}{*}{$\mathrm{Si}$} \\
\hline & HTB2 & 0,893 & 0,797 & & & & \\
\hline & HTB3 & 0.904 & 0,817 & & & & \\
\hline \multirow{3}{*}{ HTA } & HTA1 & 0,983 & 0,966 & \multirow{3}{*}{0,769} & \multirow{3}{*}{0,930} & \multirow{3}{*}{0,930} & \multirow{3}{*}{$\mathrm{Si}$} \\
\hline & HTA2 & 0,697 & 0.485 & & & & \\
\hline & HTA3 & 0,862 & 0,743 & & & & \\
\hline \multirow{3}{*}{ UTT } & UTT1 & 0.901 & 0,811 & \multirow{3}{*}{0,802} & \multirow{3}{*}{0,924} & \multirow{3}{*}{0,924} & \multirow{3}{*}{$\mathrm{Si}$} \\
\hline & UTT2 & 0,857 & 0,734 & & & & \\
\hline & UTT3 & 0,601 & 0.361 & & & & \\
\hline \multirow{3}{*}{$\mathrm{UCT}$} & UCT1 & 0,848 & 0,719 & \multirow{3}{*}{0,636} & \multirow{3}{*}{0,836} & \multirow{3}{*}{0,824} & \multirow{3}{*}{$\mathrm{Si}$} \\
\hline & UCT2 & 0,896 & 0,802 & & & & \\
\hline & UCT3 & 0,941 & 0,885 & & & & \\
\hline
\end{tabular}

Tabla 5: Resumen de resultados del modelo de medida Fuente: Elaboración propia 


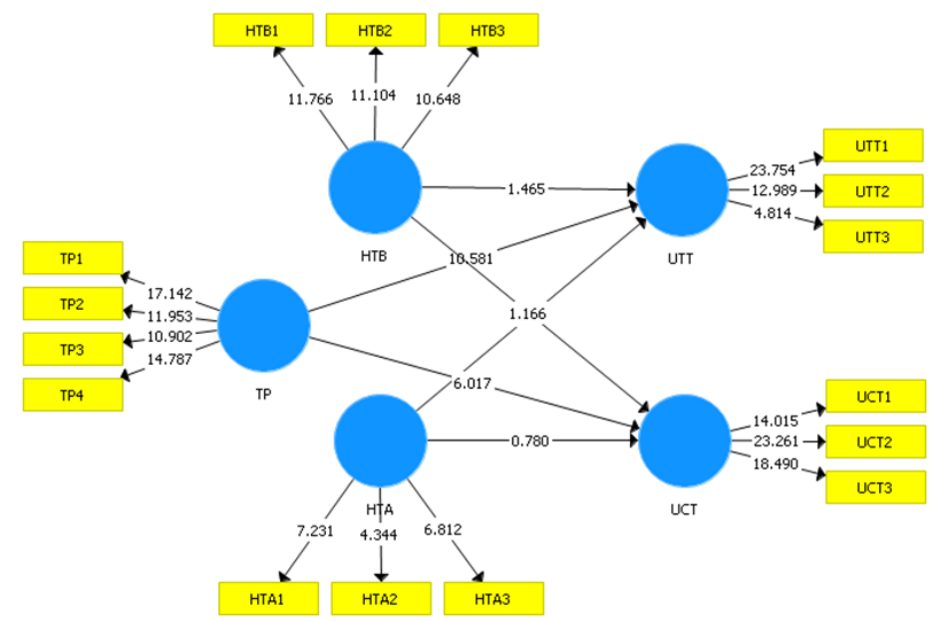

Figura 4: Resultados en el modelo usando Bootstraping

Fuente: Elaboración propia a partir de SmartPLS 3

\begin{tabular}{|l|l|l|}
\hline Variable & T Statistics & P Values \\
\hline HTA $->$ UCT & 0.780 & 0.219 \\
\hline HTA $->$ UTT & 0.362 & 0.359 \\
\hline HTB $->$ UCT & 1.166 & 0.123 \\
\hline HTB - $>$ UTT & 1.465 & 0.073 \\
\hline TP $>$ UCT & 6.017 & 0.000 \\
\hline TP $->$ UTT & 10.581 & 0.000 \\
\hline
\end{tabular}

Tabla 6: Resumen de resultados para pruebas de hipótesis Fuente: Elaboración propia

La figura 4 y la tabla 6 muestran los resultados al realizar el re muestreo y permiten obtener los estadísticos t para la prueba de hipótesis con un nivel de significancia del $5 \%$. En este caso, solo la hipótesis 5 y 6 se rechazan, luego existe una posible relación positiva significativa entre la creencia de la habilidad de usar la tecnología para la pedagogía y la intención del uso de la tecnología de forma tradicional y constructivista en el aula.

\subsection{Análisis de resultados del modelo estructural}

- Evaluación de Multicolinealidad VIF:

\begin{tabular}{|c|c|c|c|c|c|}
\hline Outer VIF Values & Inner VIF Values & & & & \\
\hline & HTA & HTB & TP & UCT & UTT \\
\hline HTA & & & & 1.916 & 1.916 \\
\hline HTB & & & & 3.046 & 3.046 \\
\hline TP & & & & 2.891 & 2.891 \\
\hline \multicolumn{6}{|l|}{ UCT } \\
\hline UTT & & & & & \\
\hline
\end{tabular}

Figura 5: Resultados de multicolinealidad

Fuente: Elaboración propia a partir de SmartPLS 3 
En la figura 5 se puede evidenciar que no hay multicolinealidad, UCT y UTT son los constructos endógenos y HTA, HTB y TP son los constructos exógenos o predictores. Como resultados, los valores de VIF son menores a 5 .

- Evaluación de la magnitud de los coeficientes path o de trayectoria: En la figura 3 se puede apreciar las magnitudes y signo de los coeficientes path o de trayectoria, en el caso de TP $>$ UTT es de 1.086, lo cual significa que hay una fuerte relación positiva entre TP y UTT. Así mismo se puede decir entre TP y UCT, ya que el coeficiente es de 0.88. Lo anterior reafirma que las hipótesis 5 y 6 se rechazan.

- Establecer la significancia de las relaciones mediante boostraping: La tabla 6 muestra los resultados de la significancia de las relaciones, las cuales permiten rechazar la hipótesis 5 y 6.

- Evaluación del coeficiente de determinación R2:

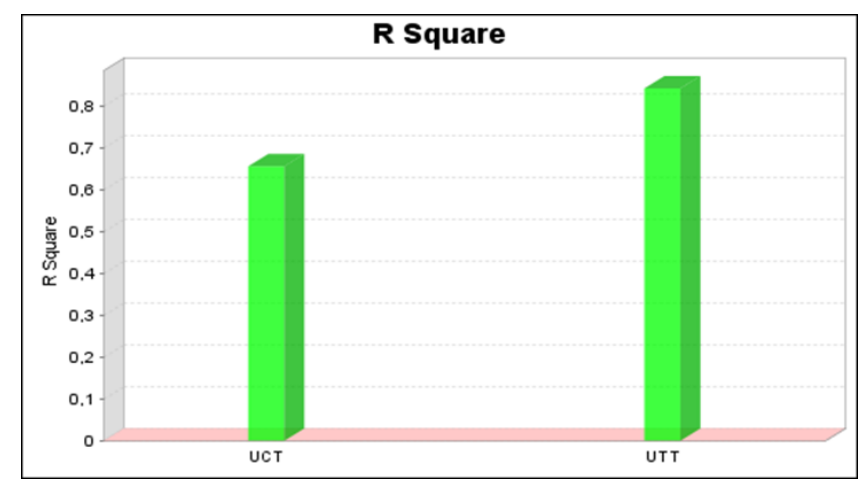

Figura 6: Coeficiente de determinación

Fuente: Elaboración propia a partir de SmartPLS 3

El coeficiente de determinación para UCT es de 0.655 y para UTT de 0.841. En el caso de UCT, el $65.5 \%$ de la varianza de UCT es explicada por los constructos endógenos HTB, HTA y TP. En el caso de UTT, el $84.1 \%$ de la varianza de UTT es explicada por HTB, HTA y TP. En otras palabras, el $84.1 \%$ implica que hay más capacidad predictiva que tiene el modelo sobre UTT, mientras que el efecto es moderado para UCT.

- Tamaño del efecto f2:

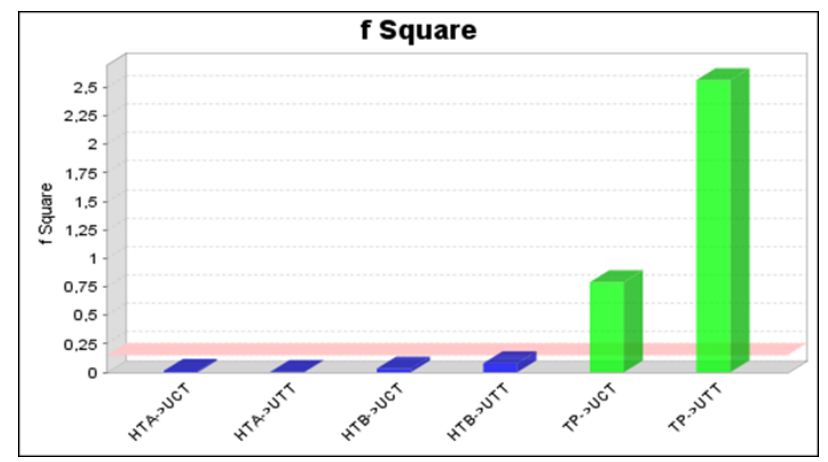

Figura 7: Grado en que el constructo exógeno (TP) explica los endógenos (UCT y UTT)

Fuente: Elaboración propia a partir de SmartPLS 3

En la figura 7 se observa como TP contribuye a explicar el constructo endógeno UCT y UTT, con valores de 0.791 y 2.564 . 


\section{Conclusiones y Recomendaciones}

- Con respecto a la pregunta general propuesta: ¿Cuál es la de las habilidades tecnológicas del docente y la intención del uso de la tecnología en la enseñanza en el aula de las universidades Tecnológica de Pereira, Quindío y Francisco José de Caldas?, se puede decir, que el modelo SEMPLS planteado evidencia una relación positiva entre el constructo del uso de la tecnología para la pedagogía y la intención del uso constructivista y tradicional en el aula, es decir, la habilidad de usar la tecnología para la pedagogía si tiene relación en la intención del uso. No existe una relación entre las habilidades tecnológicas básicas y avanzadas del uso de la tecnología y la intención en el uso, ya sea constructivista o tradicional en el aula. Este resultado se podría explicar ya que los sujetos del estudio pueden tener un conocimiento inherente de las tecnologías básicas y avanzadas descritas en el instrumento y por ende las aplican en el aula de forma habitual.

- El constructo TP o la habilidad del uso de la tecnología para la pedagogía si explica los constructos del uso intencional de la tecnología de forma constructivista UCT y tradicional en el aula UTT.

- Se podría mejorar el análisis SEM-PLS al intentar usar la técnica de multigrupo y realizar un estratificado por facultades en cada universidad y encontrar las posibles relaciones del uso de la tecnología y la intención del uso de la tecnología en el aula.

- El modelo de ecuaciones estructurales basadas en varianza presentado es evaluado mediante mínimos cuadrados parciales usando la herramienta SmartPLS, la cual es de un entorno sencillo de usar y de tratar los resultados. En R, existen múltiples librerías que están siendo construidas para realizar este tipo de análisis, sin embargo, SmartPLS permite en cuestión de pocas horas desarrollar modelos complejos.

- Posiblemente, exista sesgo en los resultados, ya que el instrumento no se replanteó las preguntas propuestas por (Teo, 2009) y se trató de evidenciar tecnologías más actuales y complejas que existan y no se estén usando por parte de los docentes, lo cual podría, ser una invitación a desarrollar un instrumento más acorde a la realidad que busque medir las complejidades de las creencias del uso de la tecnología de los docentes.

- SEM-PLS es un método que está tomando mayor fuerza en el campo de las ciencias sociales, al no ser tan restrictivo como el método de ecuaciones estructurales basadas en covarianza, se pueden desarrollar estudios con muestras relativamente pequeñas y soportados rigorosamente por conceptos o teorías que permitan plantear modelos acordes a la realidad.

- Como estudios futuros, se puede plantear estudiar el fenómeno de las creencias del uso de la tecnología, las motivaciones para usarla, la percepción de la curva de aprendizaje al aprender a usarlas y la relación con las competencias que debería tener un docente en un modelo de educación virtual. De esta forma, se podría formular estrategias para incentivar el uso de las tecnologías en el aula, el diseño de lineamientos y políticas de educación virtual que potencialicen el uso de las tecnologías en el aula y la constante preparación para apropiar las tecnologías emergentes que surjan en la actividad docente.

\section{Referencias}

Aldás Manzano, J., a. U. J. E. (2017), 'Análisis multivariante aplicado con R.', Madrid: Paraninfo .

Alvarez, O. (2002), 'La Enseñanza Virtual en la Educación Superior (1a ed.)', Recuperado a partir de http://200.116.126.171/portal/images/stories/institucional/normatcolombiana/arc 14 .pdf.

Andrews, R. \& Haythornthwaite, C. A. E. (2007), 'The Sage handbook of e-learning research (1st ed).', Los Angeles: Sage Publications. 
Barclay, D., H. C. \& Thompson, R. (1995), 'The partial least squares (PLS) approach to causal modeling: Personal computer adoption and use as an illustration.', Technology studies 2(2), 285-309.

Chambers, R. L. \& Clark, R. G. (2012), 'An introduction to model-based survey sampling with applications. Oxford', New York: Oxford University Press .

Chiu, T. K. F., . C. D. (2016), 'Adoption of mobile devices in teaching: changes in teacher beliefs, attitudes and anxiety.', Interactive Learning Environments 24(2), 317-327.

Devine, D., F. D. \& McGillicuddy, D. (2013), 'What is 'good' teaching? Teacher beliefs and practices about their teaching', Irish Educational Studies 32(1), 83-108.

Ertmer, P. A. \& Ottenbreit-Leftwich, A. T. (2010), ' Teacher Technology Change: How Knowledge, Confidence, Beliefs, and Culture Intersect', Journal of Research on Technology in Education 42(3), 255-284.

Estévez-Nenninger, E. H., V.-C. A. A.-O. C. G. \& Zavala-Escalante, M. G. (2014), 'Creencias sobre enseñanza y aprendizaje en docentes universitarios.', Magis. Revista Internacional de Investigación en Educación 6(13).

García, N., C. E. \& Ortíz, D. (2015), 'Tendencias en la Educación Virtual', Virtual Educa Red pp. 1-19.

Gutiérrez, H. A. (2009), 'Estrategias de muestreo: diseño de encuestas y estimación de parámetros', Bogotá: Facultad de Estadística, Universidad Santo Tomás. .

Hair, J. F. E. (2014), 'A primer on partial least squares structural equations modeling (PLS-SEM)', Los Angeles: SAGE. .

Hair, J. F. E. (2017), 'A primer on partial least squares structural equation modeling (PLS-SEM) (Second edition).', Los Angeles: Sage. .

Henseler, J. (2018), 'Partial least squares path modeling: Quo vadis?', Quality Quantity, 52(1), 1-8.

Hernández Sampieri, R., F. C.-C. \& Baptista Lucio, P. (2014), 'Metodología de la investigación. ', México, D.F.: McGraw-Hill Education .

Jimoyiannis, A. \& Komis, V. (2007), 'Examining teachers' beliefs about ICT in education: implications of a teacher preparation programme', Teacher Development 11(2), 149-173.

Jääskelä, P., H. P. \& Rasku-Puttonen, H. (2017a), 'Teacher Beliefs Regarding Learning, Pedagogy, and the Use of Technology in Higher Education ', Journal of Research on Technology in Education 49(3-4), 198-211.

Jääskelä, P., H. P. \& Rasku-Puttonen, H. (2017b), 'Teacher Beliefs Regarding Learning, Pedagogy, and the Use of Technology in Higher Education', . Journal of Research on Technology in Education 49(3-4), 198-211.

Kwong, K. \& Kwong, K. (2013), 'Partial Least Squares Structural Equation Modeling (PLS-SEM) Techniques Using SmartPLS', Recuperado 11 de Julio de 2016, a partir de http://marketingbulletin.massey.ac.nz/v24 $/ m b_{v} 24_{t} 1_{w}$ ong.pdf.

Lawrence, B. \& Lentle-Keenan, S. (2013), 'Teaching beliefs and practice, institutional context, and the uptake of Web-based technology', Distance Education 34(1), 4-20.

Lumpe, A. T. \& Chambers, E. (2001), 'Assessing Teachers' Context Beliefs about Technology Use', Journal of Research on Technology in Education 34(1), 93-107.

Moreno Torres, W. (1993), 'Introducción a la Inferencia Estadística. Bucaramanga.', Universidad Industrial de Santander-Colombia . 
Pérez López, C. (2014), 'Técnicas de muestreo estadistico. ', Madrid: Garceta. .

Reinartz, W., H. M. \& Henseler, J. (2009), 'An empirical comparison of the efficacy of covariance-based and variance-based SEM.', International Journal of Research in Marketing 26(4), 332-344.

Sarstedt, M., H. J. F.-R. C. M. T.-K. O. \& Gudergan, S. P. (2016), 'Estimation issues with PLS and CBSEM: Where the bias lies!', Journal of Business Research 69(10), 3998-4010.

Särndal, C.-E., S. B. \& Wretman, J. (2003), 'Model assisted survey sampling', New York: Springer .

Teo, T. (2009), 'Examining the Relationship between Student Teachers' Self-Efficacy Beliefs and Their Intended Uses of Technology for Teaching: A Structural Equation Modelling Approach. ', Turkish Online Journal of Educational Technology 8(4), 7-15. 